\title{
FRÉCHET VS. GÂTEAUX DIFFERENTIABILITY OF LIPSCHITZIAN FUNCTIONS
}

\author{
MARIA GIERALTOWSKA-KEDZIERSKA AND F. S. VAN VLECK
}

(Communicated by R. Daniel Mauldin)

\begin{abstract}
Examples have been given of Lipschitzian functions that are Gâteaux-differentiable everywhere, but nowhere Fréchet-differentiable. One such example has been reported, mistakenly, in several papers as having domain in $L^{2}([0, \pi])$, when it should have been $L^{1}([0, \pi])$. We discuss this example.
\end{abstract}

The purpose of this note is to point out a misunderstanding that has been perpetuated about an example due to Sova [7]. In order to do this, we consider two mappings: (1) $f: L^{1}([0, \pi]) \rightarrow \mathbf{R}$ defined by $f(x)=\int_{0}^{\pi} \sin x(t) d t$, and (2) $g: L^{2}([0, \pi]) \rightarrow \mathbf{R}$ defined by $g(x)=\int_{0}^{\pi} \sin x(t) d t$. Clearly, $g$ is the restriction of $f$ to $L^{2}([0, \pi]) \subseteq L^{1}([0, \pi])$. The mapping $f$ is an example of a Lipschitzian real-valued function that is everywhere Gâteaux-differentiable, but nowhere Fréchet-differentiable. In fact, $f$ is a special case of a whole class of mappings, from the space $L^{1}(X, \Sigma, \mu)$ of all $\Sigma$-measurable, $\mu$-integrable functions from $X$ to $\mathbf{R}$, defined by Sova in [7] that are Gâteaux-differentiable, but not Fréchet-differentiable.

On the other hand, the function $g$ is Fréchet-differentiable everywhere, but is given in some papers, in error, as an example of a mapping that is nowhere Fréchet-differentiable; cf. [3, p. 205], [4, p. 124], and [5, p. 125]. Earlier, Phelps [6, pp. 981-982] gave an example of an equivalent norm on $l^{1}$ that is Gâteaux-differentiable everywhere (except at the origin) and nowhere Fréchetdifferentiable. Other examples of Lipschitzian real-valued functions that are nowhere Fréchet-differentiable were given by Aronszajn in [1]; his functions are on the space $l^{1}$.

In order to consider the differentiability of $f$ and $g$, let $x, v \in L^{1}([0, \pi])$,

Received by the editors January 13, 1989 and, in revised form, August 15, 1989.

1980 Mathematics Subject Classification (1985 Revision). Primary 26E15; Secondary 26A27.

Key words and phrases. Fréchet differentiability. Gâteaux differentiability. 
$v \neq 0, h>0$. Then

$$
\begin{aligned}
\lim _{h \downarrow 0} \frac{1}{h} \int_{0}^{\pi}[\sin (x(t)+h v(t))-\sin x(t)] d t \\
\quad=\lim _{h \downarrow 0} \int_{0}^{\pi} \frac{\sin \frac{h v(t)}{2}}{\frac{h}{2}} \cos \left(x(t)+\frac{h v(t)}{2}\right) d t \\
=\int_{0}^{\pi} v(t) \cos x(t) d t,
\end{aligned}
$$

since the integrand $\left(\left(\sin \frac{h v(t)}{2}\right) /(h / 2)\right) \cos \left(x(t)+\frac{h v(t)}{2}\right)$ is dominated by $v \in$ $L^{1}([0, \pi])$. Hence, the Gâteau $x$ derivative of the mapping $f$ at $x$ is $D_{G} f(x)$ $=\cos x$. It is clear that the mapping $g$, which is the restriction of $f$ to $L^{2}([0, \pi])$, is also Gâteaux-differentiable and that the Gâteaux derivative $D_{G} g(x)=\cos x$ is a continuous mapping from $L^{2}([0, \pi])$ into $L^{2}([0, \pi])$ in the norm topologies. Therefore, $g$ is Fréchet-differentiable everywhere (see [2, Examples 1 and 2, pp. 18-22] for a more general class having the two properties described previously). Actually, in our case, $g$ is uniformly Fréchetdifferentiable. (Apply Taylor's formula to the sine function, and conclude that $|\sin (a+b)-\sin a-b \cos a|=\left|\frac{b^{2}}{2} \sin z\right|$ for some $z$ between $a$ and $a+b$. Thus,

$$
\int_{0}^{\pi}|\sin (x(t)+y(t))-\sin x(t)-y(t) \cos x(t)| d t \leq \int_{0}^{\pi} \frac{1}{2} y(t)^{2} d t=\frac{1}{2}\|y\|_{2}^{2} .
$$

Hence, if $\|y\|_{2}<2 \varepsilon$, we see that $|g(x+y)-g(x)-\langle y, \cos x\rangle| \leq \varepsilon\|y\|_{2}$.)

To prove that $f$ is not Fréchet-differentiable at any point $x \in L^{1}([0, \pi])$, we will follow Sova's proof of [7, Theorem 2.1.6]. First, we show that for each $x \in L^{1}([0, \pi])$, there exists $v \in L^{1}([0, \pi])$ such that the Lebesgue measure of the set $\{t \in \mathbf{R} \mid 0 \leq t \leq \pi$ and $\sin (x(t)+v(t))-\sin x(t)-v(t) \cos x(t) \neq 0\}$ is positive. If not, let $q$ be a rational number and define $v_{q}$ by $v_{q}(t)=q$ for all $t \in[0, \pi]$. Then $v_{q} \in L^{1}([0, \pi])$ and the set $N_{q}=\{t \in[0, \pi] \mid$ $\sin (x(t)+q)-\sin x(t) \neq q \cos x(t)\}$ has Lebesgue measure 0 . Hence, the union $N=\bigcup\left\{N_{q} \mid q\right.$ rational $\}$ also has measure 0 . Thus, for all rational numbers $q$ and all $t \notin N, \sin (x(t)+q)-\sin x(t)=q \cos x(t)$. This is a contradiction since the mapping $q \cos x(t)$ is a linear function of $q$, but $\sin (x(t)+q)-\sin x(t)$ is not.

Next, choose $v_{0} \in L^{1}([0, \pi])$ such that $\mu\left(\left\{t \in[0, \pi] \mid \sin \left(x(t)+v_{0}(t)\right)-\right.\right.$ $\left.\left.\sin x(t)-v_{0}(t) \cos x(t) \neq 0\right\}\right)>0$, where $\mu$ denotes Lebesgue measure. Then we can find $\alpha>0$ such that the set $Z=\left\{t \in[0, \pi] \mid \sin \left(x(t)+v_{0}(t)\right)-\right.$ $\left.\sin x(t)-v_{0}(t) \cos x(t)>\alpha\right\}$ satisfies $\mu(Z)>0$. Further, there exists a $\beta$ and a measurable subset $Z_{0}$ of $Z$ such that $\mu\left(Z_{0}\right)>0$ and $\left|v_{0}(t)\right|<\beta$ for $t \in Z_{0}$. Choose a sequence $\left\{Z_{n}\right\}_{n=1}^{\infty}$ of measurable subsets of $Z_{0}$ of $Z$ such that $Z_{n+1} \subset Z_{n}, \mu\left(Z_{n}\right)>0$ for $n=1,2, \ldots$, and $\bigcap_{n=1}^{\infty} Z_{n}=\varnothing$, and define a sequence $\left\{h_{n}\right\}_{n=1}^{\infty}$ of functions in $L^{1}([0, \pi])$ by

$$
h_{n}(t)= \begin{cases}v_{0}(t) & \text { if } t \in Z_{n} \\ 0 & \text { if } t \notin Z_{n}\end{cases}
$$

It is easy to check that $\left\|h_{n}\right\|_{1} \rightarrow 0$ as $n \rightarrow \infty$, but

$$
\frac{\left|\int_{0}^{\pi}\left[\sin \left(x(t)+h_{n}(t)\right)-\sin x(t)-h_{n}(t) \cos x(t)\right] d t\right|}{\left\|h_{n}\right\|_{1}} \geq \frac{\alpha \mu\left(Z_{n}\right)}{\beta \mu\left(Z_{n}\right)}=\frac{\alpha}{\beta}>0 .
$$


This shows that $f$ is not Fréchet-differentiable at $x \in L^{1}([0, \pi])$.

Added in proof. It should be noted that the authors were not the first to discover the difficulties discussed in this paper. See R. R. Phelps, Convex functions, monotone operators and differentiability, Lecture Notes in Math., vol. 1364, Springer-Verlag, New York, 1989, p. 105. See also D. Preiss, Frêchet derivatives of Lipschitz functions, J. Funct. Anal. 91 (1990), 312-345, for a very strong positive result on differentiability of Lipschitz functions.

\section{ACKNOWLEDGMENT}

The authors would like to thank the referee for pointing out Phelps' paper and the fact that $g$ is uniformly Fréchet-differentiable.

\section{REFERENCES}

1. N. Aronszajn, Differentiability of Lipschitzian mappings between Banach spaces, Studia Math. 57 (1976), 147-190.

2. J.-P. Aubin and I. Ekeland, Applied nonlinear analysis, Wiley, New York, 1984.

3. V. I. Averbukh and O. G. Smolyanov, The theory of differentiation in linear topological spaces, Russian Math. Surveys 22 (1967), 201-258.

4. J. P. R. Christensen, Topological and Borel structure, North-Holland Math. Stud., vol. 10, 1974.

5. M. Z. Nashed, Differentiability and related properties of nonlinear operators: Some aspects of differentials in nonlinear functional analysis, Nonlinear Functional Analysis (L. B. Rall, ed.), Academic Press, New York, 1971.

6. R. R. Phelps, A representation theorem for bounded convex sets, Proc. Amer. Math. Soc. 11 (1960), 976-983.

7. M. Sova, Conditions for differentiability in linear topological spaces, Czechoslovak Math. J. 16 (1966), 339-362. (Russian)

Department of Mathematics, University of Kansas, Lawrence, Kansas 66045 